\title{
El clima institucional como generador de contextos de protección y riesgo frente a las manifestaciones de violencia en centros educativos del Circuito 06 de la Dirección Regional de Educación de San José
}

\section{The institutional climate as generator of protection and risk contexts against violence in educational centers in 06 School Circuit of the San José Regional Education Bureau}

Recibido 20 noviembre 2011 • Aceptado 25 junio 2012 • Corregido 28 junio 2012

\author{
Olger Carmona Ávila ${ }^{1}$ \\ Asistente de Dirección, Ministerio de Educación Pública \\ olger.carmona.avila@mep.go.cr \\ Patricia Flores Villalobos ${ }^{2}$ \\ Docente Educación Primaria, Ministerio de Educación Pública \\ pattycr14@hotmail.es \\ Warner Gómez Araya ${ }^{3}$ \\ Docente Educación Primaria, Ministerio de Educación Pública \\ wgomez33@hotmail.com \\ Marcia Navas Alfaro ${ }^{4}$ \\ Docente Educación Secundaria, Ministerio de Educación Pública \\ abigailnavas@hotmail.com \\ Moisés Ruiz Gutiérrez ${ }^{5}$
}

1 Es Licenciado en Ciencias de la Educación con énfasis en Administración Educativa de la Universidad de Costa Rica (UCR), posee una Licenciatura en Docencia en la Enseñanza del Inglés, de la Universidad Estatal a Distancia (UNED) y un Bachillerato en la Enseñanza del Inglés de la Universidad de Costa Rica (UCR). Actualmente cursa el Posgrado en Gestión Jurídica de la Educación en la Universidad de Costa Rica y se desempeña como asistente de dirección del Colegio Técnico Profesional Abelardo Bonilla Baldares y con recargo en la Sección Técnica Nocturna del Colegio Técnico Profesional Dos Cercas, del Ministerio de Educación Pública.

2 Es Licenciada en Ciencias de la Educación con énfasis en Administración Educativa de la Universidad de Costa Rica (UCR), posee una Licenciatura en Ciencias de la Educación con énfasis en Primer y Segundo Ciclo de la Universidad Santa Lucía y un Bachillerato en Ciencias de la Educación con énfasis en Primer y Segundo Ciclo de la Universidad Nacional (UNA). Actualmente se desempeña como profesora de primaria en el centro educativo Los Pinos, Ministerio de Educación Pública.

3 Es Licenciado en Ciencias de la Educación con énfasis en Administración Educativa de la Universidad de Costa Rica (UCR), posee un Bachillerato en Ciencias de la Educación con énfasis en Educación Primaria de la Universidad de Costa Rica (UCR). Actualmente se desempeña como docente de la educación primaria en la Escuela Pio XII del Ministerio de Educación Pública.

4 Es Licenciada en Ciencias de la Educación con énfasis en Administración Educativa de la Universidad de Costa Rica (UCR), posee un Bachillerato en la Enseñanza de la Educación Física y Deportes de la Universidad de Costa Rica. Actualmente se desempeña como docente en Educación Preescolar en el Colegio María Inmaculada del Sistema Educativo Costarricense.

5 Es Licenciado en Ciencias de la Educación con énfasis en Administración Educativa de la Universidad de Costa Rica (UCR), posee una Licenciatura en Ciencias de la Educación con énfasis en Primer y Segundo Ciclo de la Universidad Santa Lucía, y un 
Director Educación Primaria, Ministerio de Educación Pública morugu@hotmail.es

Karem Smith Coto $^{6}$

Docente Educación Secundaria, Ministerio de Educación Pública smithka19@yahoo.es

Resumen. El presente artículo presenta una investigación, se plantea el objetivo general de analizar desde la gestión educativa el clima institucional como generador de contextos de protección y riesgo frente a las manifestaciones de violencia en los centros educativos del circuito 06 de la Dirección Regional de Educación de San José, Costa Rica. Aborda componentes teóricos tales como la violencia, clima institucional, modelo ecológico, organizaciones educativas y conflicto. Se utiliza un método de investigación mixto, aunado a un diseño de investigación-acción, para lo cual se trabajó con siete centros educativos del sistema educativo costarricense, pertenecientes a un mismo circuito.

Dentro de las principales conclusiones a que llegó el estudio se encuentran que el contexto hostil en el que están inmersas las instituciones educativas constituye un factor que propicia los hechos de violencia, además la gestión administrativa no promueve acciones reales que conlleven a un buen abordaje de los hechos de violencia, ya que los equipos de apoyo: equipo interdisciplinario, comités institucionales, asociaciones de padres, juntas administrativas y otros, se desempeñan de forma desarticulada. Dentro de los hechos de violencia más recurrentes destaca el bullying entre estudiantes-estudiantes, estudiantes-docentes y viceversa. Finalmente, como aporte de la investigación se diseñó una propuesta de prevención y atención a la violencia desde una perspectiva ecológica, mediante un esquema de trabajo integral, multi y transdisciplinario, que incorpora acciones a nivel comunitario, familiar y del centro educativo contribuyendo al mejoramiento de la calidad en el ambiente del aula y clima institucional.

Palabras claves. Violencia, conflicto en las instituciones educativas, conflictos estudiantiles, conflictos en las instituciones educativas, gestión de la educación, clima institucional, clima escolar, modelo ecológico.

Abstract. This paper presents analyses educational management from institutional climate in work environment as a source of protection and risk contexts of violence in schools circuit 06 of the Dirección Regional de San José (San José Regional Education Bureau), Costa Rica. This study includes theoretical components such as violence, institutional climate, work environment, ecological model, educational organizations, and conflict. The research uses an investigation mixed with method, with an investigation-action research design developed in seven schools in the Costa Rican educational system. To conclude this study, hostile environment in which educational institutions explode violence acts, and administrative management does not promote real actions to find an approach to avoid violence, as support teams: interdisciplinary, institutional committees, parent associations, and other administrative

Bachillerato en Ciencias de la Educación con énfasis en Primer y Segundo Ciclo de la Universidad Nacional (UNA). Actualmente se desempeña como director en la Escuela El Silencio del Ministerio de Educación Pública.

${ }^{6}$ Es Licenciada en Ciencias de la Educación con énfasis en Administración Educativa de la Universidad de Costa Rica (UCR), posee un Bachillerato en Enseñanza del Español de la Universidad de Costa Rica (UCR). Actualmente se desempeña como docente de la educación secundaria en el Sistema Educativo Costarricense. 
boards, they perform so disjointed. The most recurrent violence problem is bullying between students-students, students, teachers and vice versa. Finally, a proposal is presented here for preventing violence from an ecological perspective, through a comprehensive scheme of work, and multi-disciplinary, incorporating actions at community, family and the school to improve quality in the classroom environment and school climate.

Keywords. Violence, educational institutions conflict, student conflicts, educational management, school climate, work environment, ecological model.

\section{Introducción}

Los sistemas educativos se enfrentan a una serie de desafíos, según sus contextos y las necesidades de la sociedad en la cual están inmersos. Dentro de estos desafíos se encuentran factores de índole académico, social, cultural, económico que inciden en la gestión de las organizaciones educativas. Problemas de deserción, repitencia, calidad docente, infraestructura, rendimiento académico, conflictos y violencia estudiantil son unos de los retos a los que se enfrenta la administración de la educación.

La violencia se caracteriza por su complejidad; así como por la multitud de sus rasgos constitutivos, que a criterio de la Organización Mundial de la Salud (OMS), en su Informe Mundial sobre la Violencia (2002) la define como:

El uso deliberado de la fuerza física o el poder, ya sea en grado de amenaza o efectivo, contra uno mismo, otra persona o un grupo o comunidad, que provoque o tenga muchas probabilidades de causar lesiones, muerte, daño psicológico, trastornos del desarrollo o privaciones. (p. 290)

El informe de la OMS (2002) propone el Modelo Ecológico como herramienta de examen de las causas de la violencia y como enfoque de prevención. Este modelo se caracteriza por explorar la relación entre los factores individuales y contextuales y considera la violencia el producto de muchos niveles de influencia sobre el comportamiento social, comunitario, relacional e individual. Además, examina los contextos de la comunidad en los que se inscriben las relaciones sociales, la escuela, el lugar de trabajo y el vecindario, y busca identificar las características de cómo se asocian las víctimas con sus agresores, ya que esto favorece la violencia más que otros factores. Precisamente este es un campo en el que asume importancia la administración de la educación en las organizaciones educativas. 


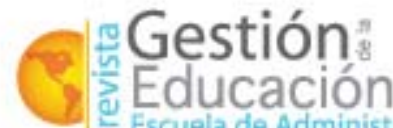 \\ Escuela de Administración Educativa}

[Número publicado el 30 de Junio del 2012]
Revista Cientifica Digital ISSN:2215-2288

URL:http:// revista dig ita l.ea e.fc s.uc r.ac.cr/

Recientes estudios nacionales, como los expuestos por Barrantes y Lara (2005); Chacón y Salas (2006), Montoya y Segura (2006), exponen la necesidad de realizar abordajes integrales del fenómeno, que incorporen: el factor institucional, la cultura institucional, el currículo, los espacios de comunicación, el diálogo, y el liderazgo de los directores y directoras de los centros educativos, los elementos de mayor peso al respecto.

Según, Arias (2009), algunas de las manifestaciones de violencia tienen su origen en la familia, en la comunidad, en la imitación de patrones de conducta y en la influencia de los medios de información de masas. Además, estos comportamientos son potenciados por el modelo curricular vigente, lo que genera resistencia a las normas institucionales.

Una línea consistente de investigación en el campo de la educación, se ha dirigido a estudiar las condiciones de riesgo y de protección que influyen sobre la violencia dado que las variables que intervienen son múltiples y complejas. Al considerar estas orientaciones, es posible deducir que una gestión de la educación efectiva, así como la generación de un clima escolar que se caracterice por una convivencia saludable y la satisfacción de sus miembros, es un campo de acción relevante para directores y directoras de la educación y el equipo directivo, ya que una convivencia escolar saludable; considerada como una construcción colectiva, histórica y dinámica, permite a sus miembros un desarrollo afectivo.

Este debe ser un tema que se aborde en forma preventiva, con el fin de evitar el posible tránsito hacia escenarios en donde, por falta de una gestión eficiente, se instalen como prácticas habituales las formas de resolución violentas de los conflictos entre los miembros de la comunidad educativa. Surge, por tanto, la pregunta ¿Cuál es la incidencia que posee el rol de las estructuras directivas en la resolución de conflictos y en las formas de abordaje de las situaciones o hechos de violencia?

Desde esta perspectiva se justifica profundizar en el estudio de este fenómeno de una manera integral, teniendo como centro el clima institucional y las prácticas educativas como eje articulador del entramado socio educativo, y su propia comunidad. El estudio del clima institucional, en tanto que es una construcción social asociada al tema de violencia, requiere considerar las percepciones de los diferentes sectores que integran la comunidad educativa.

Se propone por ello un estudio donde se utilice el modelo ecológico, que es el recomendado para este tipo de investigaciones y abordajes, además de adoptar la metodología de investigación acción participativa para que todas las personas de la comunidad educativa 
puedan expresarse, manifestarse, reflexionar y aportar al tema, para lo cual se plantearon los siguientes objetivos:

\section{Objetivos generales.}

1. Analizar desde la gestión educativa el clima institucional como generador de contextos de protección y riesgo frente a las manifestaciones de violencia en los centros educativos del circuito 06 de la Dirección Regional de Educación de San José.

2. Proponer de manera participativa líneas de acción para el fortalecimiento de competencias institucionales para prevenir y afrontar la violencia en las instituciones educativas del circuito 06 de la Dirección Regional de Educación de San José.

\section{Objetivos específicos.}

1.1 Examinar el rol de las estructuras institucionales en la resolución de conflictos y en las formas de abordaje de las situaciones o hechos de violencia en los centros educativos participantes.

1.2 Caracterizar las prácticas organizacionales de las instituciones educativas participantes desde el modelo ecológico.

1.3 Comprender las percepciones sobre conflicto y violencia que tienen los diferentes actores de la comunidad educativa.

1.4 Analizar el currículo oculto institucional como factor de riesgo o prevención de la violencia en los centros educativos.

1.5 Identificar necesidades de las instituciones educativas para fortalecer su capacidad de respuesta al fenómeno de la violencia.

\section{Marco teórico}

\section{Clima institucional y las organizaciones educativas.}

El clima institucional es definido como el conjunto de interacciones y transacciones que se generan en la tarea educativa en una situación espacio-temporal. El clima tiene un carácter 
global y es determinado por elementos de naturaleza diversa como los espacios arquitectónicos, estructura física, equipamiento, características personales de los integrantes de la comunidad educativa, sistema de relaciones establecido, sistema de valores de distintos grupos entre otros factores, el clima institucional tiene un carácter dinámico e interactivo y es susceptible de modificación (Arias, 2009).

Para analizar los factores que se manifiestan relacionados con la violencia escolar, es preciso estudiar la institución educativa en sí misma. Lo anterior, porque la violencia escolar es más vivencial a lo interno de los centros educativos por ser el contexto donde se desarrollan y convergen los educandos. Así, es preciso dedicar espacios para la definición de esta.

Un elemento clave en la configuración de la convivencia escolar es el clima institucional, por ello, no puede olvidarse en el análisis de la violencia. La micropolítica de los centros educativos, como sostiene Ball (1989), son territorios donde la influencia interpersonal, el compromiso y las negociaciones entre bastidores llegan a ser tan importantes como los procesos formales.

La mayoría de los autores mencionados consideran que un buen clima escolar se caracteriza por lo positivo de las relaciones interpersonales, por un sistema de reglas y normas claras y coherentes en su aplicación y por un nivel bajo de victimización, intimidación o maltrato, pero un centro educativo que ignora e implícitamente permite los comportamientos violentos es un centro en el cual su rasgo esencial es la competitividad y el individualismo que provocan un clima de violencia institucional al no saber prevenir y resolver los conflictos de manera pacífica (Johnson y Johnson, 1999).

Viñas y Domenech (2001), indican que hay seis diferentes climas en una organización y que estos dependen de las prácticas organizativas existentes. De esta manera proponen, los siguientes:

1. Clima relacional: hace referencia al ambiente que reina en las relaciones entre los distintos miembros de la comunidad educativa en su dimensión socioafectiva, dependiendo de la bondad de los contactos interpersonales, del respeto entre todos los miembros de la comunidad educativa y del apoyo mutuo prestado (comunicación).

2. Clima educativo positivo: procesos de enseñanza y aprendizaje, actividad del profesorado, integración entre el alumnado y programa de estudios. Afirman los autores, que está probada la influencia de los aspectos organizativos de los centros "sobre todo 


\section{Gestión: \\ Escuela de Administración Educativa}

[Número publicado el 30 de J unio del 2012]
Revista Cientifica Digital ISSN:2215-2288

URL:http:// revista dig ita l.ea e.fc s.uc r.ac.cr/

cuando se desarrolla en su interior de manera que el clima educativo definido explícitamente en todo proyecto curricular, como proyecto colectivo, no es ajeno a las coordinadas organizativas en las que este se desarrolla".

3. La organización interviene, como elemento mediador entre la enseñanza y el aprendizaje, de manera que determina cualquier acción que se realice en ese ámbito

4. Clima de seguridad: remite al orden y a la tranquilidad necesarios para que se produzca en las mejores condiciones, el proceso de enseñanza-aprendizaje, favoreciendo los buenos sentimientos y la confianza entre todos y procurando reducir los factores de riesgo que conducen a la victimización, el maltrato o la vulneración de los derechos más elementales.

5. Clima de justicia: reglas y normas básicas de convivencia y de disciplina escolar. Un clima escolar justo es aquel en que se reconoce la legitimidad y equidad de las reglas, aplicadas sin discriminación y sin arbitrariedad, valorándose el mérito de cada cual, así como su dignidad personal.

6. Clima de pertenencia: es aquel que permite la cohesión de los grupos y favorece los lazos estables de los miembros de la comunidad educativa, especialmente del alumnado, con el centro educativo. El clima de pertenencia está relacionado especialmente con el grado y calidad de cohesión interna de un centro educativo y con la capacidad que este tiene para implicar a todos sus miembros de manera responsable en un proyecto educativo previamente acordado. (p. 33)

Por su parte, Janosz (1998) propone que el estudio de las prácticas del clima institucional es relevante en el análisis de la violencia en centros educativos y se deben integrar como práctica lo siguiente: reglamento escolar, resultados académicos, calidad y los tiempos dedicados a la enseñanza; actividades escolares y extraescolares, participación de la familia, liderazgo y estilo de la gestión directiva. Además, Janosz (1998) considera que estas prácticas educativas están determinadas por los siguientes elementos:

1. Comportamiento esperado en el cumplimiento de las normas de convivencia.

2. La organización física del aula.

3. Las habilidades comunicativas del profesorado.

4. El tipo de intervención del profesorado ante el comportamiento del alumnado. 


\section{Gestión: \\ S. Educación \\ Escuela de Administración Educativa}

[Número publicado el 30 de J unio del 2012]
Revista Cientifica Digital ISSN:2215-2288

URL:http:// revista dig ita l.ea e.fc s.uc r.ac.cr/

5. La comprensión del modo de adaptación emocional y social del alumno.

6. La autonomía del alumno. (p.20)

\section{Violencia, conflictos y dimensiones.}

En la presente investigación, se considera de relevancia la definición de la violencia que ofrece el Fondo de las Naciones Unidas para la UNICEF $(2006$, p.6) al ser considerada como " (...) un fenómeno que ejerce alguien sobre otro (s) coartando su libertad; es una acción donde se somete intencionalmente al maltrato a los demás". La violencia se manifiesta de diferentes categorías y es por esta razón que ofrece diversas acepciones. Dentro de estas se encuentra la violencia física y emocional. Este tipo de violencia se realiza desde las personas adultas con la intención de disciplinar, para corregir o cambiar una conducta no deseable y sustituirla por conductas socialmente aceptables y que las personas adultas consideran importantes para el desarrollo de la niñez y la adolescencia. Es el uso de la fuerza causando dolor físico o emocional a la persona agredida.

Ortega (1997) propone tres variables a estudiar en el comportamiento violento en las escuelas:

1. Dimensión evolutiva: relacionada con el proceso de desarrollo sociomoral y emocional de estudiantes.

2. Dimensión psicosocial: tipo de relaciones con sus iguales en el proceso de socialización que afecta a los aspectos socioafectivos de las relaciones interpersonales dentro de las comunidades y grupos de pertenencia o vínculo y en procesos de convivencia que ofrece el medio escolar.

3. Dimensión educativa: estilos de enseñanza, los modelos de disciplina escolar, los sistemas de comunicación y de organización.

La violencia presenta formas variadas de manifestaciones, cada una obedece a una en particular, pero al final el resultado repercute en el mismo. Los conflictos son introductorios para lo que serán las formas de violencia. Dicho de otra manera, se puede afirmar que los actos de violencia tienen como punto de origen los conflictos. Los conflictos son muy diversos, y se pueden presentar por razones muy variadas. Sin embargo, lo que sí resulta homogéneo a la 
hora de estudiar los conflictos es que, el trato no apropiado de los enfrentamientos repercute de manera directa en un acto de violencia.

El conflicto es considerado por Thomas, citado por Robbins (2004, p. 395) como "un proceso que comienza cuando una parte percibe que otra afectó o va a afectar algo que le interesa". A su vez, existe una gama extensa de conflictos que tienen las personas en las organizaciones dentro de los que se destacan la incompatibilidad de metas, diferencias en la interpretación de los hechos, desacuerdos sobre las expectativas de comportamiento que van desde los actos descarados y violentos hasta las formas más sutiles de desacuerdo.

Con respecto de las razones por las que se originan los conflictos se presentan variadas razones, por ejemplo las ofrecidas por la Fundación Arias para la Paz y el Progreso Humano (2003), quienes exponen como razones originadoras de conflictos las siguientes:

- Falta de interés (evasión).

- Adopción de conductas inaceptables (discriminación, intolerancia, falta de respeto).

- Información equivocada (rumores).

- Desinformación.

- Información inexacta (incompleta, contradictoria, compleja, perturbadora, mala interpretación).

- Rencores personales.

- Enojo (sentimientos negativos). (p. 91)

\section{Factores relacionados con la violencia escolar desde el punto de vista de la teoría ecológica.}

La violencia es un fenómeno complejo, multicausal y multifactorial, esta condición exige que su abordaje se dé bajo los más óptimos estándares de estudio. Por lo que se considera adecuada la teoría ecológica (específicamente del modelo ecológico) como guía para el estudio del fenómeno de la violencia.

Hace unas décadas, el psicólogo estadounidense Bronfenbrenner, citado por FríasArmenta (2006, p.35) mantenía que "la mayor parte de la psicología evolutiva de la época hacía referencia a la conducta extraña de los niños en situaciones extrañas con adultos extraños". Por su parte, Lewin, citado Frías-Armenta (2006) fue uno de los pioneros que mantuvo que la 
conducta surge en función del intercambio de la persona. Lo que afirma los postulados de Bronfenbrenner de tener en cuenta que el ambiente "ecológico" se circunscribe al sujeto, esta situación llevó a Bronfenbrenner, citado por Frías-Armenta (2006) a considerar que:

(...) el desarrollo humano como una progresiva acomodación entre un ser humano activo y sus entornos inmediatos (también cambiantes). Pero este proceso, además, se ve influenciado por las relaciones que se establecen entre estos entornos y por contextos de mayor alcance en los que están incluidos esos entornos. (p. 48)

De esta forma el autor propone una perspectiva ecológica del desarrollo de la conducta humana. Esta expectativa manifiesta que el ambiente ecológico es una escala estructural que se constituye por varios niveles de orden individual, microsistema, mesosistema y macrosistema. Estos distintos ambientes definidos en el modelo ecológico son a su vez sistemas, funcionando como tales, en los cuales el ser humano es un elemento más. Dentro de estos sistemas, los aspectos físicos entendidos como vivienda, configuración de un barrio, ruido, son también elementos en interacción que han de ser considerados en la valoración e intervención comunitaria.

Por su parte, los modelos ecosistémicos describen los procesos adaptativos e inadaptativos de las personas y los factores situacionales e individuales que median en esos procesos. La aportación de Dohrenwend, citado por Villalba (2003), se aproxima a una comprensión con visión ecosistémica de los procesos relacionados con la inadaptación. Al respecto, se elaboró un modelo conceptual de inadaptación que se apoya en el concepto de tensión psicológica, y no necesariamente psicopatológica, frente a acontecimientos vitales estresantes. Además, consideró el crecimiento psicológico como un posible resultado ante la respuesta al estrés. Para una mayor comprensión del modelo ecológico empleado como sustento teórico en la presente investigación, se ilustra en la Figura 1 cada uno de sus niveles, que son los factores y contextos determinantes en la investigación propuesta, el cual incluye la multiplicidad de los factores que podría asociarse al origen a la violencia. Cabe mencionar que, por la relevancia del modelo ecológico y por la facilidad que aporta para el estudio desde diferentes fenómenos, presenta la particularidad y la oportunidad de ser empleado para el análisis de cualquier evento asociado a la temática. Lo único que se debe tener en cuenta es que para poder hacerlo efectivo, se debe ajustar al contexto del fenómeno en estudio. Por lo 
demás, la funcionalidad y practicidad del modelo queda en evidencia una vez que es puesto en marcha.

Figura 1

Modelo ecológico por Urie Bronfenbrenner

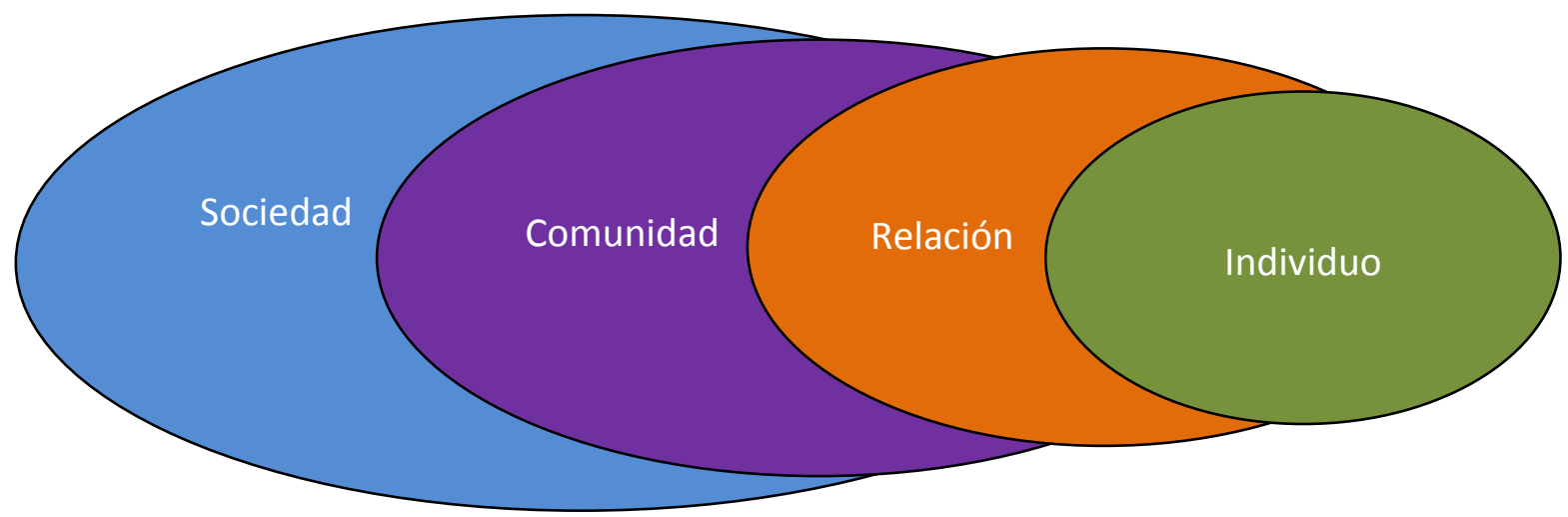

Fuente: OMS, (2003).

El centro de estudio o de interés para este modelo es el individuo. Toda su historia y características propias tienen gran relevancia en su desarrollo y comportamiento. Desde esta óptica el contexto en el cual vive y se desarrolla el individuo incluye factores situados a diversos niveles, más o menos cercanos y que ejercen influencias directas y/o indirectas.

El microsistema constituye el nivel más inmediato en el que se desarrolla el individuo, usualmente la familia y las relaciones que se generan en este. El mesosistema comprende las interrelaciones de dos o más entornos en los que la persona en desarrollo participa activamente visto en plena en la comunidad donde el ser humano convive. Por último, el macrosistema lo configuran la cultura y la subcultura en la cual se desenvuelve la persona y todos los individuos de su sociedad.

\section{Metodología de la investigación}

Al tratarse de una investigación social, se decidió manejar un método mixto. Esto implicó la aplicación de un diseño cualitativo y cuantitativo de forma secuencial e independiente. Es decir, se inició con una fase cualitativa, esta sirvió de base para diseñar la fase cuantitativa para 
luego terminar con otra fase cualitativa. Los datos obtenidos de estas fases se complementan y así se llegó a las conclusiones y a la construcción de la propuesta.

El objetivo de fase cualitativa es analizar de manera conjunta el problema de la investigación desde la visión y percepción de los sujetos de la investigación y con base en ello; diseñar la fase cuantitativa, sirviendo este último estudio como método de triangulación de la investigación.

Se aplicó el diseño de investigación-acción; puesto que, interesaba la reflexión sobre el problema y avanzar por aproximación sucesiva en la comprensión del fenómeno de la violencia a partir de la perspectiva de los actores fundamentales.

La fase cualitativa se desarrolló en los centros educativos del circuito educativo 06, y para esta se organizaron tres momentos de reflexión-acción. En el marco de la fase cuantitativa, la técnica de recolección de información fueron tres cuestionarios sobre percepción de violencia en el ámbito escolar, convivencia, estilos de solución de conflicto, y clima organizacional aplicado a estudiantes, docentes y directores/directoras de los centros de estudio.

Como resultado del proceso de reflexión- acción, se trabajó con siete centros educativos interesados en seguir el proceso hasta el final. Estos centros educativos fueron: Escuela Carolina Dent, Escuela Carmen Lyra, Escuela de Tejarcillo, Escuela Los Pinos, Escuela Hatillo 2, Liceo Teodoro Picado; así como, el Kínder Hatillo 8.

Como muestra se seleccionó dos secciones por cada nivel educativo, desde el segundo ciclo de educación general básica hasta la educación diversificada. Siguiendo el Modelo Ecológico, se aplicó un cuestionario a todos los docentes de esas mismas secciones, así como al director de la institución. De esta manera la población estuvo conformada por 572 estudiantes, siete directores de los centros educativos y 100 docentes, se trabajó con la totalidad de ellos.

Las técnicas de recolección de información empleadas consistieron en tres momentos de reflexión-acción, correspondientes a la fase cualitativa, y la aplicación de tres cuestionarios sobre percepción de violencia en el ámbito escolar, convivencia, estilos de solución de conflicto y clima organizacional aplicado a estudiantes, docentes y directores de los centros de estudio, como parte de la fase cuantitativa.

Para el análisis de datos se empleó la escala Alfa de Cronbach, coeficiente que sirve para medir la fiabilidad de una escala de medida, y la escala de Chi Cuadrado, prueba que mide la independencia o relación de dos variables entre sí. 


\section{Análisis de resultados}

En general hay una alta percepción de la violencia, la convivencia en el centro educativo se ha ido deteriorando, y las sanciones adoptadas han sido poco efectivas; no obstante cabe destacar que en el Liceo Teodoro Picado, la mayoría de los docentes estiman que la violencia en su institución y comunidad es baja y que las medidas adoptadas han sido adecuadas, por lo que los niveles de convivencia han mejorado.

Los centros educativos cuyos docentes perciben un alto nivel de violencia son: Escuela Carmen Lyra, seguida de la Escuela Carolina Dent. En el caso de la institución de Tejarcillo, a pesar que es poca la diferencia entre quienes perciben altos niveles y bajos niveles de violencia, la gran mayoría $(70,2 \%)$ estima que el nivel de convivencia se ha deteriorado, pese a que casi el (67\%) opina de las sanciones y medidas adoptadas por la institución han sido las correctas.

El clima organizacional, es un elemento clave en la configuración de la convivencia escolar, por ello, no puede olvidarse en el análisis de la violencia. Investigaciones previas como las de Tuvilla (2004); Johnson y Johnson (1999) y Ortega (1997) han demostrado consistentemente como factor protector o como factor de riesgo al clima organizacional. A los docentes se les realizó tres preguntas, la primera de ellas es general, siendo una pregunta sobre la relevancia del clima con la situación de violencia, acto seguido se ejecutó dos preguntas sobre esta variable en su institución, una de ellas como factor de riesgo, y la otra como factor de protección.

De acuerdo con los resultados, el $63 \%$ de los docentes consultados estiman que el clima organizacional es muy relevante como factor de riesgo o protección de los hechos de violencia. Como se observa los datos del Chi cuadrado son significativos:

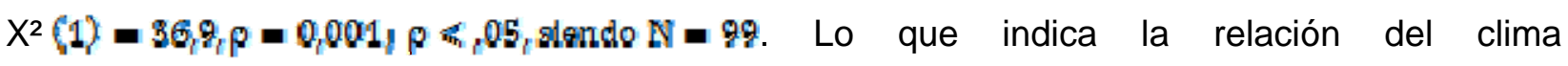
organizacional con los hechos de violencia en la institución.

La valoración que hacen del clima organizacional de su centro educativo es también, consistente con los resultados anteriores. Para realizar el análisis se sumaron las alternativas de "a menudo" con la de "siempre", para formar una nueva variable de "sí lo considera factor de riesgo". De esta manera, los resultados indican que el clima es considerado como factor de riesgo por un significativo porcentaje de docentes de Tejarcillo (48\%) y de Hatillo $2(44,4 \%)$. 


\section{Giestion: \\ Escuela de Administración Educativa}

[Número publicado el 30 de J unio del 2012]
Revista Cientifica Digital ISSN:2215-2288

URL:http:// revista digital.eae.fc s.ucr.ac.cr/

Para la variable: el clima como factor protector o que minimiza los hechos de violencia, se sumaron las variables de "nunca" con la de "pocas veces", para formar una nueva "no lo minimiza", para relacionarlo con la pregunta anterior. Así, los resultados son consistentes, en Tejarcillo, el $(47,4 \%)$ no considera que lo minimice y en esa misma dirección Hatillo 2 con un $(44,4 \%)$. Llama la atención que una importante mayoría $(58,8 \%)$ de los docentes del Centro Educativo Carolina Dent, le atribuyen al clima organizacional la contención a los hechos de violencia en la institución, de la misma manera lo estima la Escuela Carmen Lyra con un (75\%) de docentes.

Queda claro que el clima representa un papel de gran importancia como factor de riesgo y/o protección frente a los hechos de violencia en las instituciones. A partir de estos resultados es donde se debe preparar a la comunidad educativa para dar énfasis en el aspecto protector, ya que si los docentes, alumnos y demás actores del proceso educativo sienten una alta identificación con el centro, será más fácil la colaboración para afrontar las situaciones de violencia en las instituciones.

También, a partir de este hecho crear un plan institucional de contención de la violencia que realmente sea acorde a la realidad de cada comunidad para lograr mayor efectividad, en donde participen docentes y alumnos de manera activa durante el proceso.

Los centros educativos como organización, son espacios de convivencia caracterizados por las interrelaciones entre sus miembros, reguladas por normas básicas de organización y funcionamientos (Tuvilla, 2004). El clima institucional, es un elemento clave en la configuración de la convivencia escolar, por ello, no puede olvidarse en el análisis de la violencia, la micropolítica de los centros educativos, ya que como sostiene Ball (1989), son territorios donde la influencia interpersonal, el compromiso y las negociaciones entre bastidores llegan a ser tan importantes como los procesos formales.

La gran mayoría de los niños, niñas y adolescentes consultados viven con su madre $(77,36 \%)$ y hermanos $(51,62 \%)$. Con el padre la cantidad es apenas del $(26,54 \%)$. Lo anterior configura un tipo de familia predominantemente uniparental con presencia de otros familiares. Es importante mencionar, la presencia de padrastros y madrastras (25\%) y un (21\%), respectivamente.

A manera de síntesis, se puede afirmar que, desde el punto de vista del estudiante y en el marco de análisis del modelo ecológico, una cuarta parte de los estudiantes de las 
instituciones participantes del estudio se encuentran en situación de alta vulnerabilidad, en tanto ni su grupo familiar, ni la escuela le representan un factor de protección y contención.

Normalmente en la cultura de una organización existen estilos predominantes en el abordaje de los conflictos, líneas de solución habituales a los mismos, y un contexto de mayor o menor regulación formal de las decisiones que afectan a la convivencia. El estilo de resolución de conflicto está vinculado al clima organizacional y este como factor protector o facilitador de los hechos de violencia en los centros educativos, de ahí la importancia de conocer los estilos del personal directivo a cargo de las instituciones en estudio.

El instrumento de evaluación de la modalidad de conflictos ha sido diseñado para evaluar el comportamiento de un individuo en situaciones de conflicto.

Figura 2

Estilos de resolución de conflictos de directores de centros estudiados

\begin{tabular}{lll}
\hline Dimensión & $\begin{array}{l}\text { Asertividad } \\
\text { (individuo trata de } \\
\text { satisfacer sus } \\
\text { necesidades e } \\
\text { intereses) }\end{array}$ & $\begin{array}{l}\text { Cooperatividad } \\
\text { (individuo intenta satisfacer los } \\
\text { intereses y necesidades de } \\
\text { otras personas) }\end{array}$ \\
\hline Alto & $\begin{array}{ll}\text { Competitividad } \\
\text { Medio }\end{array}$ & $\begin{array}{l}\text { Acomodarse } \\
\text { (Directores 1, 2 y 6) }\end{array}$ \\
Cojo & $\begin{array}{l}\text { Compromiso (Directores 3, } 4 \text { y 7 ) } \\
\text { Acomodarse }\end{array}$ & Competitividad \\
& Evitación & \\
(Director 5) & \\
\hline
\end{tabular}

Fuente: Elaboración propia con base en resultados estadísticos del estudio (2011).

\section{Resultados de la fase cualitativa.}

La información se sistematizó de acuerdo con las preguntas generadoras que fueron trabajadas durante el taller realizado en el mes de octubre de 2010. Estas preguntas tenían como objetivo conocer el interés en el abordaje del tema sobre el clima institucional como generador de protección y riesgo frente a manifestaciones de violencia, relevancia de la 
investigación sobre el tema, aspectos y dimensiones cruciales de analizar en el problema de violencia en centros educativos. Esta información se presenta en la siguiente tabla:

\section{Tabla 1}

Factores de influencia en los jóvenes, justificación de interés en el tema y aspectos y dimensiones cruciales de análisis en centros educativos

\begin{tabular}{|c|c|}
\hline $\begin{array}{l}\text { - La sociedad: cultura, } \\
\text { ambiente. } \\
\text { Influye en el estado de } \\
\text { ánimo }\end{array}$ & $\begin{array}{ll}\text { - } & \text { La televisión sustituye a los padres } \\
\text { - } & \text { No hay límites } \\
\text { - } & \text { La escuela es vista como una guardería } \\
\text { - } & \text { Los padres no saben qué hacer con sus hijos } \\
\text { - } & \text { El sitio no debe influir en la crianza, esto debe ser algo muy personal } \\
& \text { para poder salir adelante en un ambiente hostil } \\
\text { - } & \text { Los compañeros influyen grandemente en el comportamiento } \\
\text { - } & \text { El ambiente donde se vive influye, pero la familia es un gran apoyo }\end{array}$ \\
\hline - La familia & $\begin{array}{l}\text { - Violencia familiar: papá le pega a mamá, hermanos contra hermanos } \\
\text { - Niños abusados } \\
\text { - } \quad \text { Niños golpeados } \\
\text { - } \quad \text { La familia es el principal ente de violencia } \\
\text { país } \\
\text { - La pobreza influye }\end{array}$ \\
\hline - Lucha de poderes & $\begin{array}{l}\text { - Estudiantes pelean por saber quién es el más fuerte: conducta contraria } \\
\text { en sus casas, pues allí son sumisos } \\
\text { - Existe un trasfondo evidente: la familia: hay una lucha, se representa } \\
\text { esa imagen violenta (fortaleza) para no ser violentado }\end{array}$ \\
\hline $\begin{array}{l}\text { - Costa Rica es un } \\
\text { país cada vez más } \\
\text { violento }\end{array}$ & $\begin{array}{l}\text { La violencia ha estado presente siempre, solo que antes eran hechos } \\
\text { más privados, se escondían, ahora son más públicos y se dan en todos } \\
\text { los lugares. Costa Rica, en general, presenta un ambiente más } \\
\text { violento. }\end{array}$ \\
\hline $\begin{array}{l}\quad \text { Las } \\
\text { instituciones } \\
\text { educativas como } \\
\text { factor a investigar. }\end{array}$ & $\begin{array}{l}\text { - Cultura institucional rígida e irrespetuosa hacia la opinión de } \\
\text { estudiantes } \\
\text { - Instituciones muy limitadas en cuanto a recursos, por lo que es muy } \\
\text { difícil disponer de espacios y de actividades estimulantes hacia } \\
\text { estudiantes } \\
\text { - } \quad \text { Algunos docentes también son violentos en las aulas } \\
\text { - Oferta extracurricular limitada o nula }\end{array}$ \\
\hline
\end{tabular}

Fuente: Taller desarrollado con directores y personal docente de los centros educativos participantes del estudio (2010).

El problema principal fue delimitado de manera conjunta y negociado en este mismo taller. Quedó claro que no era un programa del Ministerio de Educación Pública, sino que, se quería saber cómo está la institución educativa colocada frente al problema de la violencia en los centros escolares, dado que sobre el particular muy poco se tenía información. 
Figura 3

Resumen Visión Integral del Modelo Ecológico

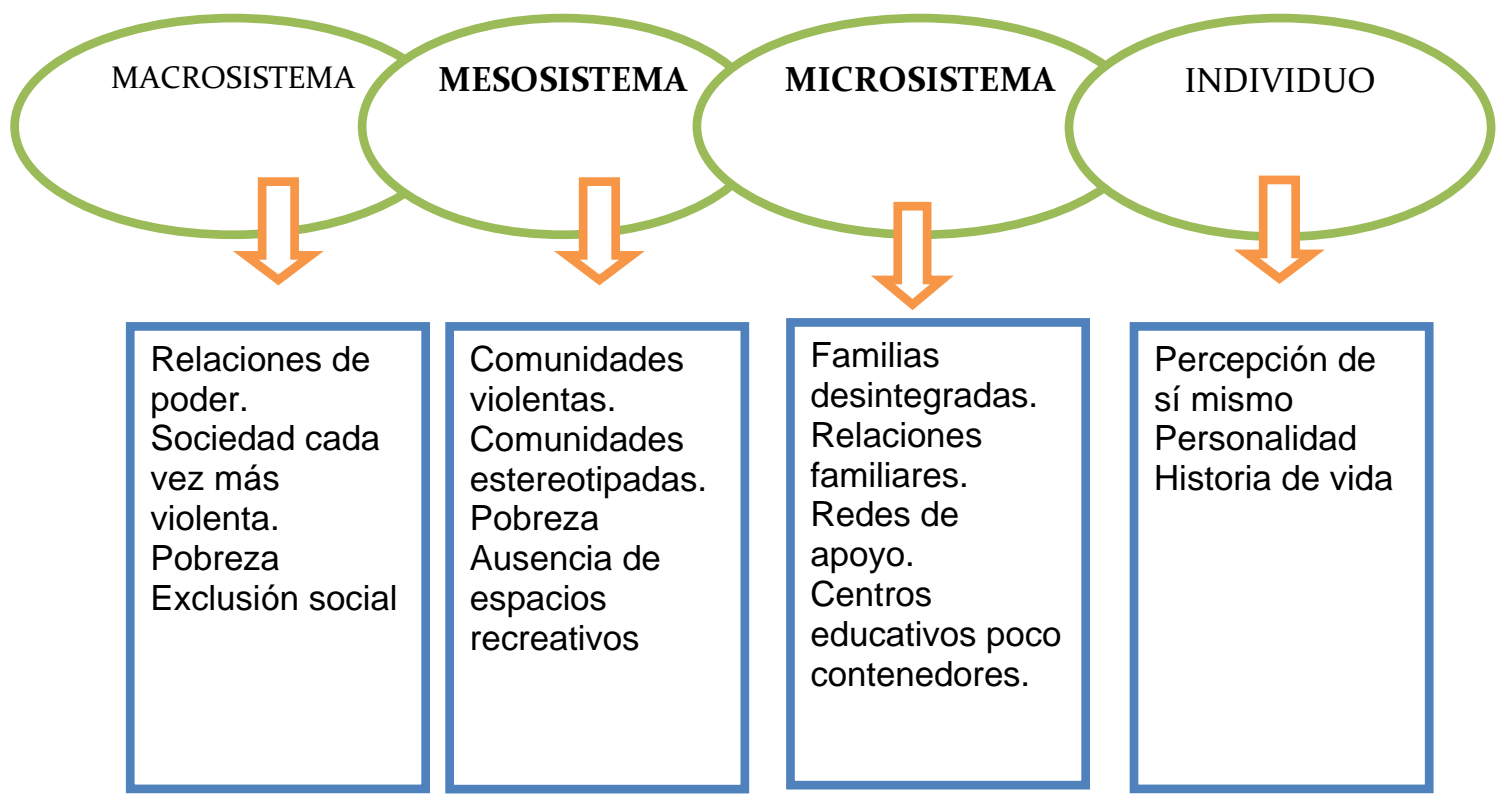

Fuente: Elaboración propia con base en datos de la presente investigación (2011).

Cada nivel del modelo ecológico se constituye en una unidad de análisis a partir del conjunto de resultados obtenidos tanto en la fase cuantitativa como en la cualitativa.

\section{Propuesta: prevención y atención de la violencia}

Los datos obtenidos en el proceso de la investigación reflejan que los agentes sociales que participan del proceso de enseñanza aprendizaje de esta zona, están inmersos en un ambiente con índices considerables de violencia, pobreza y exclusión social.

En relación con el ambiente familiar la mayoría son familias monoparentales, en otras se da la presencia de padrastros y también, no en pocos casos, varias familias comparten una misma vivienda. Ante estas situaciones, un porcentaje importante de estudiantes reportan una mala calidad en la relación familiar, especialmente los colegiales. Más de una cuarta parte de los estudiantes manifiestan vivir en un clima familiar conflictivo y violento. 


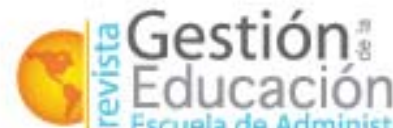 \\ Escuela de Administración Educativa}

[Número publicado el 30 de J unio del 2012]
Revista Cientifica Digital ISSN:2215-2288

URL:http:// revista dig ita l.ea e.fc s.uc r.ac.cr/

Los docentes perciben un ambiente hostil, muy violento, tanto en su comunidad como en el centro educativo donde laboran. La mayoría de ellos y ellas atribuyen como causa de la violencia, a la violencia intrafamiliar y a las condiciones sociales en las cuales viven sus estudiantes. Ante esta realidad, también se externa por parte del personal de las instituciones educativas, que no están completamente preparados para abordar temas de violencia y califican de deficiente el apoyo del Ministerio de Educación.

Cabe mencionar que el clima institucional se percibe como generador y facilitador de actos de violencia, o sea que no es un factor de protección ni reducción de actos de violencia, pues no existe una visión sistémica, ni ecológica para el abordaje de la violencia en los centros educativos.

Específicamente, en cuanto a los cargos de dirección se refiere, sobresale que la gran mayoría presentan estilos de resolución de conflictos que lo evitan o lo enmascaran, pero no lo abordan de manera directa y asertiva. Esto es un factor importante que podría incidir en un clima de convivencia no saludable.

Por tanto, se requiere el planteamiento de un esquema de trabajo integral, multi y transdisciplinario, que incorpore acciones a nivel comunitario, familiar y del centro educativo.

Al ser la violencia una problemática que se ha generalizado en todo el planeta, es comprensible encontrar distintas propuestas y proyectos programados en varios países, para la prevención y atención de la violencia.

En todos los programas que se tomaron en cuenta como antecedentes para la elaboración de esta propuesta, se evidencian puntos en común que se deben tomar en cuenta para la prevención y abordaje de la violencia.

Los entornos o sistemas de atención:

- Individual: El desarrollo personal de los estudiantes requiere del apoyo de todos quienes les rodean, incluyendo sus pares, o sea, entre ellos mismos.

- Familiar: Es el primer ambiente de aprendizaje de las y los jóvenes. Ahí se dan las bases de la formación del individuo, el desarrollo de los estudiantes, pues en ese lugar permanecen gran cantidad de horas.

- Comunitario: Es el ambiente inmediato de la institución educativa. Se valora como factor de riesgo o de protección según sea el caso. 


\section{G Gestión

[Número publicado el 30 de J unio del 2012]
Revista Cientifica Digital ISSN:2215-2288

URL:http:// revista dig ita l.ea e.fc s.uc r.ac.cr/

Además, las prácticas educativas y la gestión escolar tiene un impacto extraordinario sobre el clima relacional (sobre todo entre el profesorado y el equipo directivo de los centros y el profesorado entre sí), así como en el clima educativo y el clima de pertenencia. Algunos elementos que no se pueden pasar por alto son:

- El comportamiento esperado en el cumplimiento de las normas de convivencia.

- La organización física del aula.

- Las habilidades comunicativas del profesorado.

- El tipo de intervención del profesorado ante el comportamiento del alumnado.

- La comprensión del modo de adaptación emocional y social del alumnado.

- La autonomía del alumno.

- La participación de las familias.

Tomando en cuenta los antecedentes, se formula una propuesta de intervención y prevención al fenómeno de la violencia para su implementación en centros educativos del Circuito 06 de la Dirección Regional de San José, llamada: Gestión de un Centro Seguro.

La prevención y la atención del problema de la violencia atañen a todos los agentes sociales participantes del proceso educativo, tanto estudiantes, familiares, miembros de la comunidad y entidades estatales, principalmente la institución educativa, que es la entidad directamente relacionada con todos los demás elementos del sistema. Se puede decir que la institución educativa es el enlace con el resto de sectores; de ahí la importancia que se da al liderazgo y al clima.

\section{La prevención.}

De acuerdo con las investigaciones realizadas, las estrategias de prevención de la violencia en la institución educativa, se hace particularmente desde el hogar. Si bien es cierto que en la institución educativa también se trabaja la prevención; es el hogar el primer ambiente en que se desarrolla la y el estudiante, por ende es de gran importancia dotar a los miembros de las familias con estrategias de convivencia armoniosa, combatir la violencia y fundamentar la necesidad de crear ambientes propicios al desarrollo integral y saludable del estudiante. 


\section{Gestión \\ Sìnducación \\ Escuela de Administración Educativa}

[Número publicado el 30 de J unio del 2012]
Revista Cientifica Digital ISSN:2215-2288

URL:http:// revista digita l.eae.fc s.ucr.ac.cr/

El abordaje.

Este se hace cuando emerge una situación violenta dentro de la institución educativa. Para tal abordaje se aplica un protocolo para resolución de conflictos en donde se evidencie la mediación y la conciliación si fuese el caso. En esta parte del plan de acción, se hace necesario el conocimiento por parte del personal del centro educativo, el seguimiento que se hace de los casos que van aflorando. Esto con el fin de respetar el debido proceso y no incurrir en la indefensión de alguno de los niños, niñas, jóvenes y/o adultos involucrados.

Figura 4

Esquema Gestión Centro Seguro

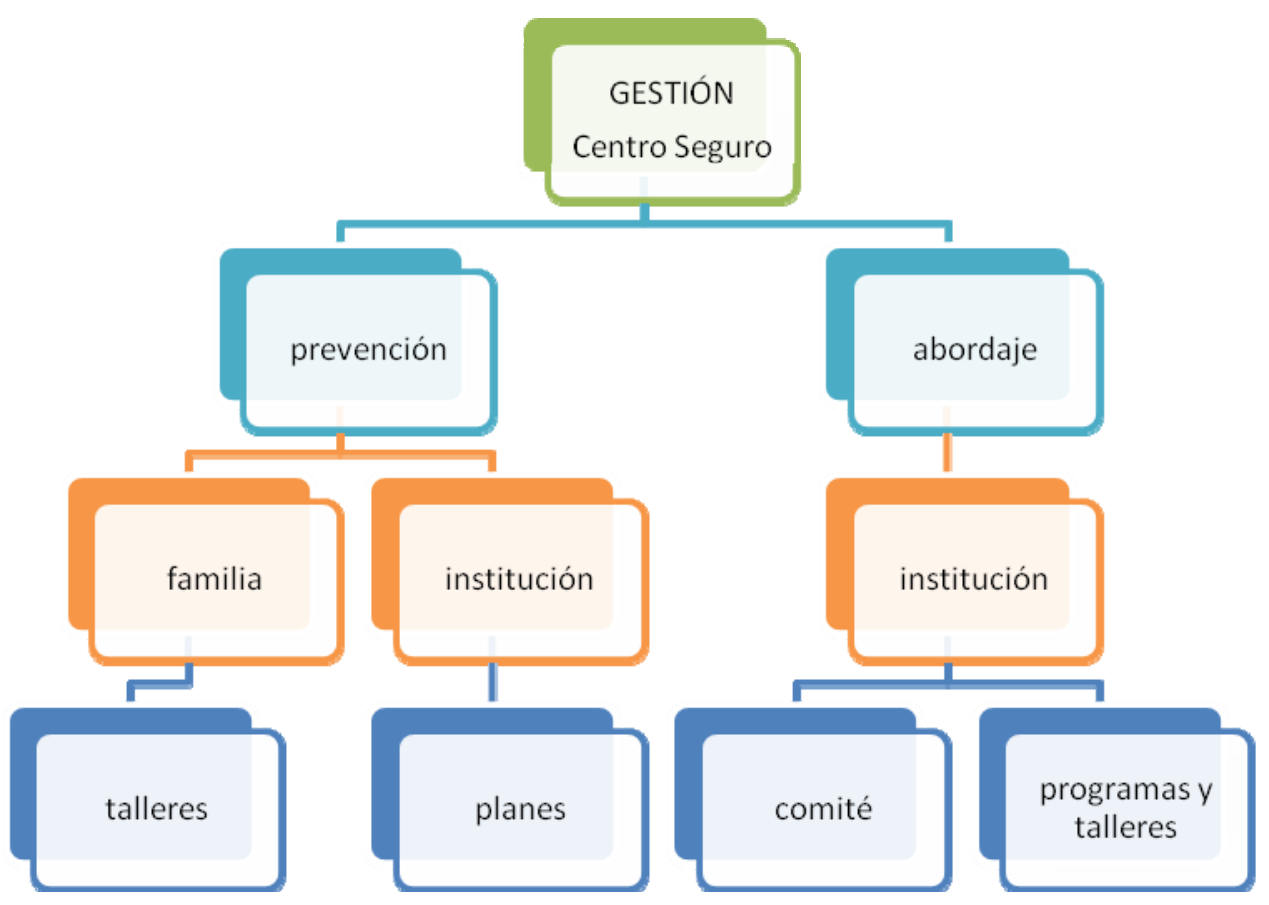

Fuente: Elaboración propia (2011).

\section{Gestión de un centro educativo seguro.}

En adelante se conocerá como "centro educativo seguro", a aquella institución educativa que ofrezca un ambiente apropiado para la sana convivencia. Para comenzar con este proyecto 


\section{Gestión:

[Número publicado el 30 de J unio del 2012]
Revista Cientifica Digital ISSN:2215-2288

URL:http:// revista dig ita l.ea e.fc s.uc r.ac.cr/

se sugieren las siguientes estrategias. Estas se basan tanto en estudios previos como en los resultados obtenidos en la presente investigación.

La prevención e intervención se ponen en práctica mediante talleres, capacitaciones y estrategias definidas por cada centro educativo en consideración a las especificidades de cada contexto y población. Es importante que se tome en cuenta a todos los actores sociales involucrados con el centro educativo en aras de propiciar intervención y resultados que respondan a soluciones ecológicas, es decir, que todos los ambientes y microambientes se vean beneficiados por las acciones realizadas.

\section{Toma de conciencia.}

Esta parte de sensibilización empieza desde los centros educativos, los que inducirán a los padres de familia a la toma de conciencia, de la necesidad de prevenir y resolver los problemas de la violencia que se den en el centro educativo.

Dado el alto grado de pasividad de la comunidad educativa esta tarea deberá ser realizada por la Dirección del centro, o por algún grupo de profesores y padres especialmente comprometidos. Por ejemplo, los miembros del Equipo interdisciplinario, en aquellas instituciones donde los hay, o bien el Comité de la No Violencia que debe funcionar en cada institución educativa.

Aunque en alguno de los Centros, este problema no fuese muy evidente, es necesario que esté preparado para afrontarlos, y no acudir a la improvisación. Se logrará mediante charlas y talleres en el primer mes del curso lectivo.

\section{Definición del problema.}

Hay que diferenciar, dentro de los comportamientos que no son deseables en los alumnos, distintos niveles de gravedad, de manera que podamos definir bien el problema y limitar su alcance. A cada situación le corresponde una respuesta.

- Brusquedad, malos modos, gritos, insultos, expresiones soeces: Por desgracia se ha generalizado en nuestra sociedad un cierto deterioro de los modales. Son desagradables, pero no podemos considerarlos actos de violencia. 
- Falta de disciplina: Perturbación del orden o conducta disruptiva. Obstaculizan la eficacia educativa, pero tampoco son en la mayoría de los casos actos de violencia, y requieren un tratamiento específico.

- Impulsividad: En niños pequeños se da con cierta frecuencia, y es un problema que ha de ser observado atentamente. Es una dificultad para controlar la propia conducta, que puede necesitar el tratamiento de un especialista (déficit de atención, trastornos de hiperactividad). Conviene tratarlo cuanto antes porque provoca retraso escolar y un mal ajuste social. Además, si no se trata, favorece la aparición de conductas agresivas.

- Carácter iracundo: Hay alumnos con un estilo afectivo propenso a la furia. Son susceptibles, se encolerizan con facilidad. En muchas ocasiones el mismo chico puede sentir miedo de sus propias reacciones. Los autores antiguos decían que la furia es una locura breve. No conduce a un acto violento cuando se tiene capacidad de autocontrol. Los programas de educación emocional pueden enseñar a controlar la ira.

- Actos de violencia: Es el comportamiento dirigido a hacer daño (físico o psicológico) a otra persona, aprovechándose de la superioridad física o de la ayuda de otros. Incluiría actos de crueldad con animales, actos de vandalismo y robos. Este comportamiento suele ir acompañado de otras conductas antisociales, como consumo excesivo de alcohol o de otras drogas, actos delictivos, sexo precoz o promiscuo, desprecio a toda forma de autoridad, entre otros.

Dentro de los actos de violencia podemos incluir el acoso o bullying. Olweus (1986 y 1991) define las situaciones de acoso e intimidación cuando al alumno es agredido o se convierte en víctima también, cuando está expuesto, en forma constante y durante un tiempo prolongado, a acciones negativas que lleva a cabo otro alumno o un grupo de ellos.

A su vez, define las acciones negativas cuando alguien, de forma deliberada, causa un daño, hiere o incomoda a otra persona, lo que implica la definición de conducta agresiva (Olweus, 1973). Se entiende que el acoso no es sino la repetición de actos de violencia o la amenaza de estos.

Para comprender el problema, una vez diferenciados unos comportamientos de otros, es necesario conocer la situación general de la convivencia en el centro. Sobre esto coinciden muchos de los expertos: la prevención de la violencia escolar tiene una dimensión social, formada por las relaciones que se dan dentro del colegio y en la comunidad a la que este 


\section{Q: Gestión \\ Escuela de Administración Educativa}

[Número publicado el 30 de J unio del 2012]
Revista Cientifica Digital ISSN:2215-2288

URL:http:// revista digital.eae.fc s.ucr.ac.cr/

pertenece. Todo ello, junto con las características del entorno familiar de cada alumno, forma el "clima social" en el cual conviven los estudiantes.

La relación entre las familias y la escuela es un punto fundamental. Como prevención general para cualquier tipo de conflictos es importante mantener una comunicación fluida entre padres y profesores (en este caso, a través de quien esté a cargo del Comité de No Violencia.

En casos de violencia escolar habría que mantener la privacidad de las familias, solucionar los problemas teniendo en cuenta lo que será mejor para el alumno y asegurarse de que los padres y los profesores tienen suficiente información sobre el problema.

La propuesta presenta, a su vez, una serie de recomendaciones para ejecutar desde todos los ambientes generadores o protectores de hechos violentos: la familia, la institución, la comunidad, el estado y la universidad. Esto con el fin de tratar este fenómeno de la violencia mediante el despertar de una conciencia colectiva.

\section{Conclusiones}

El (32,58\%) de los docentes entrevistados sí considera que dichas estructuras institucionales son de riesgo. Estos resultados se reflejaron en dos instituciones educativas en particular; así se puede apreciar en la tabla de resultados.

Respecto de las formas de abordaje y de acuerdo con los resultados encontrados se concluye que casi un (40\%) de los entrevistados no tienen equipos de apoyo. Además, las estructuras institucionales asumen de manera parcial y sin un claro programa institucional los acontecimientos de violencia en las instituciones. Lo anterior incluye una falta de vinculación entre lo que sucede y lo propuesto en el proyecto de Centro.

Los equipos interdisciplinarios están desarticulados de la gestión directiva, es decir, hay una falta de coordinación efectiva que logre abordar el problema de la violencia en la institución. Tal desvinculación (equipos interdisciplinarios - gestión directiva - estructuras institucionales) es evidente en la percepción que tienen los docentes de su propia preparación en el abordaje de la violencia. Tales consideran que se sienten muy poco preparados o capacitados para el abordar de manera efectiva la situación de violencia cada vez que esta se presenta en su labor. Además, y como dato relevante, notar que los docentes reflejan en sus respuestas una responsabilidad directa por parte del Ministerio de Educación como el encargado de tal ausencia de conocimiento y capacitación. 
En cuanto a la precepción que tienen los estudiantes acerca del rol de las estructuras institucionales, ellos identifican conductas violentas en los docentes y demás personal de muy diferentes formas, a saber: un alto porcentaje de quienes intimidan, insultan y ridiculizan. Aunque, según los docentes la mayoría de sus estudiantes presentan conductas violentas, estos, los estudiantes, también así perciben a sus educadores lo que convierte esta situación en todo un ciclo de violencia, el cual se refuerza consciente o inconscientemente debido que ante tales actos estudiantiles, los maestros igualmente responden con violencia.

A nivel general, es innegable que el clima institucional y los niveles de convivencia no son los adecuados para promover espacios sanos de no violencia, pues los altos porcentajes que arrojaron los resultados en contra de las estructuras institucionales muestran que este es un factor que promueve el desarrollo de la violencia.

Respecto, al estilo de resolución de conflictos y el rol que juegan las estructuras institucionales al respecto, se concluye que un (42\%) de los entrevistados prefieren ajustarse a lo que presenta el clima institucional; el otro $(57,1 \%)$ de estos entrevistados consideran que el estilo que ven presente en su institución no es el adecuado para resolver el problema. Es decir, la conciencia de que dichas estructuras están fallando es algo palpable en la respuesta de los docentes y que por lo tanto es necesario dar el paso siguiente para la solución a dicha problemática: la acción.

De acuerdo con los resultados arrojados es necesario que cada institución educativa regule la convivencia que incentiva los estados violentos en la organización ofreciendo y fortaleciendo espacios que contrarresten dichos estados impulsivos; ante lo cual es fundamental el rol de la administración de la educación en las organizaciones educativas, con el propósito de operacionalizar los fines y objetivos de la educación costarricense.

Otro dato es que los docentes consideran que la participación en gobiernos estudiantiles es deficiente, y este así como otras actividades costumbristas en centros educativos son modelos agotados que a la postre someten a toda una institución en la monotonía y por tanto los lleva a estados de estrés que incentivan la violencia. Por tanto, es menester buscar opciones que reflejen una solución eficaz, moderna y atractiva al estudiante.

Entre las actitudes y actos de violencia que se manifiestan en los centros educativos, se evidencia que la discriminación, burlas y amenazas (bullying) son las acciones que se presentan con más frecuencia y casi todos los días. Diferente sucede con los actos donde se 
presentan situaciones de golpes, robos, gritos, destrucción de útiles escolares, los cuales son los apartados que registran menos índice de incidencia.

Igualmente, sobre este tema es destacable los altos registros que presenta una institución educativa en específico, la cual muestra que estos actos son más comunes ahí versus las otras organizaciones tomadas en cuenta en esta investigación.

El (67\%) de los docentes encuestados expresa que existen diferencias entre las normas que aplican; en relación con las ejercidas por sus colegas, es decir, no existe uniformidad entre lo que se desarrolla a nivel de centro educativo como proyecto institucional, lo que hace más complicado la mecánica de atención de los actos de violencia.

Al mismo tiempo, la mayoría de los docentes manifiesta impulsar la participación en cooperativo de sus estudiantes, es decir, que la mayoría de los trabajos académicos en el aula son desarrollados de manera cooperativa donde los subgrupos trabajan en conjunto para alcanzar los objetivos y buscan la integración de los discentes dentro de las actividades que desarrollan en las lecciones que imparten.

La violencia se traduce en acciones que cometen los estudiantes y existe una alta percepción de las muestras de violencia según lo expresan los docentes a los que se les aplicó el instrumento. Dentro de las principales causas los docentes expresan que es la violencia intrafamiliar la causante principal de la violencia que manifiestan los estudiantes.

Los docentes expresan que son las amenazas lo que ellos generalmente manifiestan inconscientemente a sus estudiantes, como una manera de aplacar dichos actos. Es su propio sistema de acción.

Estos mismos, como se ha dicho anteriormente, consideran que requieren de mayor capacitación y fortalecimiento de habilidades para abordar el fenómeno de la violencia; así como una constante actualización en talleres para afrontarla.

Por su parte, se considera que los trámites administrativos que llevan a cabo más todas las labores docentes les demanda mucho tiempo, situación que los vuelve susceptibles a caer en actividades no efectivas en el abordaje de la violencia.

Además, los administradores de la educación son conscientes de que se apegan muy rígidamente a la normativa lo que les impide abrir espacios a la búsqueda de una solución integral para abordar la violencia.

De esta manera, las principales conclusiones desde el modelo ecológico, son las siguientes: 
- Los centros educativos del Circuito 06 transcurren en una zona con altos índices de violencia, pobreza y exclusión social. El entorno social no favorece el desarrollo integral de las personas menores de edad, los índices de criminalidad son muy altos, siendo además una comunidad altamente estigmatizada.

- Prevalecen las familias monoparentales y presencia de padrastros. Los estudiantes reportan en un porcentaje importante una mala calidad en la relación familiar, especialmente de los colegiales.

- Más de una cuarta parte de los estudiantes manifiestan vivir en un clima familiar de conflicto.

- Existe una alta percepción de violencia por parte de los docentes, tanto de su comunidad como de su centro educativo.

- Docentes atribuyen como causa de la violencia a la violencia intrafamiliar, y a las condiciones sociales en las que viven sus estudiantes.

- El personal consultado de los centros educativos manifiestan no estar completamente preparados para abordar temas de violencia y califican de deficiente el apoyo del Ministerio de Educación.

- Clima institucional es generador y facilitador de actos de violencia, no es un factor de protección ni reducción de actos de violencia.

- No existe una visión sistémica, ni ecológica para que se aborde la violencia en los centros educativos.

- Los directores en su gran mayoría presentan estilos de resolución de conflictos que lo evitan o lo enmascaran, pero no se aborda de manera directa y asertiva. Esto es un factor importante que podría incidir en un clima de convivencia no saludable.

- No hay procedimientos claros de cómo enfrentar hechos de violencia. Confunden la indisciplina con violencia.

- El accionar de los equipos interdisciplinarios es percibido por docentes como desarticulados a la gestión integral del Centro.

- Se requiere de manera urgente un esquema de trabajo integral, multi y transdisciplinario, que incorpore acciones a nivel comunitario, familiar y del centro educativo. 
En definitiva, se recomienda mayor participación del Estado, en este caso del Ministerio de Educación Pública en capacitación a través de talleres o conversatorios para que los docentes de estas instituciones puedan abordar de manera eficaz las situaciones de violencia que se presentan en sus instituciones. De esta forma, la administración de la educación asume importante responsabilidad, liderando en forma competitiva la gestión de los procesos institucionales de manera que se alcancen los propósitos establecidos según la política institucional.

\section{Referencias bibliográficas}

Arias, L. (2009). "Una mirada al fenómeno de la violencia escolar en Costa Rica". Revista Electrónic@ Educare. Recuperado de http://www.una.ac.cr/educare/PDF/04-PDF-V_XIIIN1_2009/06-ARIAS-UNA_MIRADA.pdf.

Ball, S. (1989). La micropolítica de la escuela. Hacia una teoría de la organización escolar. Barcelona, España: Paidós.

Barrantes, F. y Lara, D. (2005) Los factores escolares y familiares generadores de violencia. (Trabajo final de graduación para optar al grado de Licenciatura en Educación Primaria). Universidad de Costa Rica, San José, Costa Rica.

Chacón, A. y Salas, S. (2006). Fundamentos teóricos-metodológicos de los equipos interdisciplinarios en Escuelas de Atención Prioritaria de PROMECUN para el abordaje de las Manifestaciones de Violencia Escolar. (Trabajo de graduación para optar por el grado de Licenciatura en Trabajo Social). Universidad de Costa Rica, San José, Costa Rica.

Fondo de Naciones Unidas para la Infancia (UNICEF). (2006). La violencia contra niños, niñas y adolescentes. (Informe de América Latina en el marco del estudio mundial de las Naciones Unidas). Recuperado de http://savethechildrendominicana.org/pdf/estudio_ violencia_lam.pdf

Fondo de Naciones Unidas para la Infancia (UNICEF). (2006). Prevención del abuso infantil. Recuperado de www.unicef.org/.../protection_8079.htm-

Frías-Armenta, M. (2006). Predictores de la conducta antisocial juvenil: un modelo ecológico. Brasil: Red Estudios de Psicología.

Fundación Arias para la Paz y el Progreso Humano. (2003). Los poderes de la negociación. San José, Costa Rica: Autor.

Janosz, M. (1998). "El ambiente socioeducativo de la escuela secundaria: un modelo teórico para guiar la evaluación". Revue Canadiene du Psycho Education, pp. 105-125.

Johnson, D. y Johnson, R. (1999). ¿Cómo reducir la violencia en las escuelas? Buenos Aires, Argentina: Paidós.

Montoya, A. y Segura, S. (2006). Construyendo alternativas para la atención de la violencia en los centros educativos. La experiencia en la Escuela Cocorí de Cartago. (Trabajo de graduación para optar por el grado de Licenciatura en Trabajo Social). Universidad de Costa Rica, San José, Costa Rica.

Olweus, D. (1973). Personality and aggression. En: J.K. Cole, \& D.D. Jensen (Eds.), Nebraska Symposium on Motivation 1972. Lincoln: University of Nebraska Press. 
Olweus, D. (1986). Mobbning - vad vi vet och vad vi kan göra. Stockholm: Liber.

Olweus, D. (1991). Bully/victim problems among schoolchildren: Basic facts and effects of a school based intervention program. En D.J. Pepler y K.H. Rubin (Eds.), The development and treatment of childhood aggression, pp. 411-448. Hillsdale, NJ: Erlbaum.

Organización Mundial de la Salud (OMS). (2002). Informe Mundial sobre la violencia y la salud. Ginebra: Organización Mundial de la Salud (OMS).

Ortega, R. (1997). "El proyecto Sevilla anti-violencia escolar. Un modelo de intervención preventiva contra los malos tratos entre iguales". Revista de Educación, 313, pp. 143158. Recuperado de: http://dialnet.unirioja.es/servlet/articulo?codigo=19148

Robbins, S. (2004). Comportamiento Organizacional. Conceptos, controversias y aplicaciones. México, D.F.: Prentice Hall Hispanoamérica.

Tuvilla, J. (2004). Convivencia escolar y resolución de conflictos. Recuperado de http/www.educacionenvalores.org/spip.php?article1530

Villalba Q., C. (2003). "Redes Sociales: un concepto con importantes implicaciones en la intervención comunitaria". Revista Intervención Psicosocial, 2(4), pp. 69-85. Recuperado de: http://www.copmadrid.org/webcopm/publicaciones/social/1993/vol1/indice.htm

Viñas, J. y Domenech, J. (2001). El sistema relacional en la organización de centros educativos. Aspectos básicos. Barcelona, España: Praxis educativa. 